\title{
Potential of Biosynthesized Silver Nanoparticles as Nanocatalyst for Enhanced Degradation of Cellulose by Cellulase
}

\author{
Bipinchandra K. Salunke, Shailesh S. Sawant, Tae Koo Kang, \\ Deok Yun Seo, Youngjong Cha, Sun A. Moon, Bassam Alkotaini, \\ Ezhaveni Sathiyamoorthi, and Beom Soo Kim \\ Department of Chemical Engineering, Chungbuk National University, Cheongju, Chungbuk 362-763, Republic of Korea \\ Correspondence should be addressed to Beom Soo Kim; bskim@chungbuk.ac.kr
}

Received 9 January 2015; Revised 13 February 2015; Accepted 13 February 2015

Academic Editor: Zhenhui Kang

Copyright (C) 2015 Bipinchandra K. Salunke et al. This is an open access article distributed under the Creative Commons Attribution License, which permits unrestricted use, distribution, and reproduction in any medium, provided the original work is properly cited.

Silver nanoparticles (AgNPs) as a result of their excellent optical and electronic properties are promising catalytic materials for various applications. In this study, we demonstrate a novel approach for enhanced degradation of cellulose using biosynthesized AgNPs in an enzyme catalyzed reaction of cellulose hydrolysis by cellulase. AgNPs were synthesized through reduction of silver nitrate by extracts of five medicinal plants (Mentha arvensis var. piperascens, Buddleja officinalis Maximowicz, Epimedium koreanum Nakai, Artemisia messer-schmidtiana Besser, and Magnolia kobus). An increase of around twofold in reducing sugar formation confirmed the catalytic activity of AgNPs as nanocatalyst. The present study suggests that immobilization of the enzyme onto the surface of the AgNPs can be useful strategy for enhanced degradation of cellulose, which can be utilized for diverse industrial applications.

\section{Introduction}

Silver nanoparticles (AgNPs) have been extensively used in many commercial products. They are important components for research on biomedicine, optics, electronics, magnetics, mechanics, catalysis, energy science, and so on $[1,2]$. The processes of biological synthesis of AgNPs are simple, inexpensive, and environmentally benign and do not use high amount of energy and sophisticated instruments in the synthesis protocols unlike physicochemical methods [2]. Use of ecofriendly reducing and capping agents in biological synthesis route makes the synthesized AgNPs as less-toxic, biocompatible, and useful for biomedical applications [25]. Plant extracts have been demonstrated to be promising reducing and capping agents for biosynthesis of variety of metal nanoparticles such as silver, gold, and copper nanoparticles [6-8].

Efficiency of electron transfer of AgNPs is better than gold nanoparticles in biosensors. The metal nanoparticle and biomolecule interactions have received increasing attention in recent years for the development of diagnostics, sensors, and targeted drug delivery. Immobilization of glucose oxidase on AgNPs was demonstrated to increase sensitivity of glucose biosensors by three times [9]. Many organic syntheses of complex molecules utilize nanoparticles for catalysis based on their exceptional catalytic activities and selectivity to materials. The size and shape of nanoparticles, preparation conditions, addition of support materials, and the capping agent play critical role in catalysis [10].

Cellulose is the most abundant organic polymer on Earth [11]. Cellulose gives structural integrity to the primary cell wall of green plants, many forms of algae, and the oomycetes. Cellulose can be broken down through hydrolysis to smaller polysaccharides like cellodextrins or completely into glucose units. However, hydrolysis of cellulose is relatively difficult compared to other polysaccharides because cellulose molecules bind strongly to each other. Enzymes are biological catalysts that speed up reactions in the presence or absence of cofactors without any change in their activity. The enzyme cellulase performs hydrolysis of cellulose. Cellulases 
are chiefly produced by fungi, bacteria, and protozoa, which hydrolyze 1,4- $\beta$-D-glycosidic linkages in cellulose, hemicellulose, lichenin, and cereal $\beta$-D-glucans. This process has commercial applicability in food processing, textile industries, laundry detergents, pulp and paper industries, and pharmaceutical industries. Cellulases also have promise in the fermentation of cellulosic biomass into biofuels.

Metal nanoparticles have been demonstrated as beneficial catalysts in industry. Nickel nanoparticles were used as a catalyst for the chemoselective oxidative coupling of thiols to disulfides [12]. Polyvinylpyrrolidone- (PVP-) capped silver nanoparticles were used for the oxidative coupling of $n$ dodecanethiol, $n$-butanethiol, and $n$-octanethiol to their corresponding disulfides [13]. High yield and turnover number were reported after the use of silica-supported AgNPs as solid and recyclable catalysts for Diels-Alder cycloadditions of $2^{\prime}$ hydroxychalcones and dienes [14]. Starch hydrolysis using amylase was enhanced after the use of AgNPs as catalysts [15]. The reduction of dyes by sodium borohydride occurred due to the catalytic properties of AgNPs supported on silica spheres [16].

The present study aims to investigate potential of Korean traditional medicinal plants for the synthesis of AgNPs and utility of plant synthesized AgNPs in enzyme-catalyzed cellulose hydrolysis. The nanoparticle-bimolecular interaction could be potentially useful in diverse industrial applications.

\section{Materials and Methods}

2.1. Chemicals and Reagents. Cellulase (Viscozyme, Sigma, USA), carboxymethyl cellulose (CMC, Sigma, USA), silver nitrate (Samchun pure chemicals, Republic of Korea), and all other chemicals were of high purity. Ultra-filtered MilliQ water (Millipore, USA) was used for all the experiments.

2.2. Preparation of Extracts from Medicinal Plants. Medicinal plant materials (Mentha arvensis var. piperascens, Buddleja officinalis Maximowicz, Epimedium koreanum Nakai, Artemisia messer-schmidtiana Besser, and Magnolia kobus) were purchased from local medicinal plant market of Cheongju, Korea, and dried for 2 days at room temperature. The plant extracts were prepared by boiling $10 \mathrm{~g}$ of each plant material in $200 \mathrm{~mL}$ of sterile distilled water for 15 minutes. The extracts were filtered through vacuum filtration assembly using Whatman number 1 filter paper. The filtered plant extracts were immediately used for the synthesis of AgNPs.

2.3. Synthesis and Time Course Studies of Silver Nanoparticles. Typically, $25 \mathrm{~mL}$ of leaf extract was added to $475 \mathrm{~mL}$ of $2 \mathrm{mM}$ aqueous precursor $\mathrm{AgNO}_{3}$ solution for reduction of $\mathrm{Ag}^{+}$in an Erlenmeyer flask for a reaction. The reaction was allowed to occur at temperature of $55^{\circ} \mathrm{C}$ in shaking incubator at $175 \mathrm{rpm}$ for 24 hours. The reduction of pure $\mathrm{Ag}^{+}$was monitored by measuring the UV-visible spectrum between 200 and $800 \mathrm{~nm}$ wavelengths of the reaction medium using UV-visible spectrophotometer (UV-1601, Shimadzu, Japan). Time courses of synthesis of AgNPs by plant extracts were monitored by taking absorption spectra at absorption maxima of AgNPs synthesized by each plant extract in UVvisible spectrophotometer.

2.4. Characterization of Silver Nanoparticles. The AgNPs synthesized by plants were purified by repeated centrifugation at $15,000 \mathrm{rpm}$ for 20 minutes followed by redispersion of the pellet in deionized water. The purified dried powder of silver nanoparticles was further analyzed by electron dispersive Xray spectroscopy (EDS, Philips XL-30). Morphology of the silver nanoparticles was characterized by scanning electron microscopy (SEM, Philip model CM 200) and X-ray photoelectron spectroscopy (XPS) using an ESCALAB 210 with an Al X-ray source $(1486.6 \mathrm{eV})$ and analyzer. Transmission electron microscopy (TEM) micrographs were obtained using energy filtering transmission electron microscope (JEM-2100 F, HR, Jeol Ltd.) operating at $200 \mathrm{kV}$.

2.5. Quantification of Reducing Sugars upon Degradation of Cellulose by Cellulose. Tests were performed in tubes containing CMC as a substrate and plant synthesized AgNPs. Free CMC solution without AgNPs was used as control. Fungal $\beta$-glucanase units $\geq 100\left(\sim 1.2 \mathrm{~g} / \mathrm{mL}\right.$ at $\left.25^{\circ} \mathrm{C}\right)$ of cellulase (Viscozyme, Sigma, USA) were used to hydrolyze cellulose. CMC $(10 \mathrm{mg} / \mathrm{mL})$ was added in separate tubes containing $5 \mathrm{~mL}$ distilled water and autoclaved at $121^{\circ} \mathrm{C}$ for 15 minutes. The tubes were cooled to bring temperature of the solutions at $25^{\circ} \mathrm{C}$. To these separate tubes, different plant synthesized nanoparticle solutions $(5 \mathrm{~mL})$ were added and $500 \mu \mathrm{L}$ Viscozyme was added to each tube. The reactions were carried out at $25^{\circ} \mathrm{C}$ in shaking incubator and formation of sugar (glucose) was monitored in microtiter plates at $500 \mathrm{~nm}$ using glucose assay kit (Bio Clinical System Co., Ansan, Republic of Korea). The amount of glucose generated was estimated from standard graph of glucose.

\section{Results and Discussion}

The solution of $\mathrm{AgNO}_{3}$ after addition of plant extracts turned pale yellow and then to brown. This colour change indicated the synthesis of AgNPs (Figure 1(a)). The plasmon peaks for the AgNPs synthesized by M. arvensis, B. officinalis, E. koreanum, A. messer-schmidtiana, and M. kobus were observed at 450,434,438,421, and $416 \mathrm{~nm}$, respectively. The occurrence of plasmon peaks between 400 and $500 \mathrm{~nm}$ confirmed the presence of AgNPs as reported earlier [47]. The formation of AgNPs was increased with increase in incubation time. E. koreanum showed rapid synthesis of AgNPs among different plant extracts (Figure 1(b)).

EDS measurement showed presence of $\mathrm{Ag}$ in all the samples of AgNPs synthesized by plants (Figure 2(a)). The highest Ag content in terms of weight $\%$ and atom \% was found for M. kobus and lowest for M. arvensis (Table 1). The Ag $3 d_{5 / 2}$ and Ag $3 d_{3 / 2}$ core level binding energies for AgNP samples in XPS appeared at 368 and $374 \mathrm{eV}$, suggesting presence of metallic silver in the samples. A representative EDS and XPS image for AgNPs is presented in Figure 2(b).

SEM micrographs showed presence of nanoparticles in all the samples (Figure 3). The AgNPs were monodispersed in nature. The size of the particles from TEM image ranged 


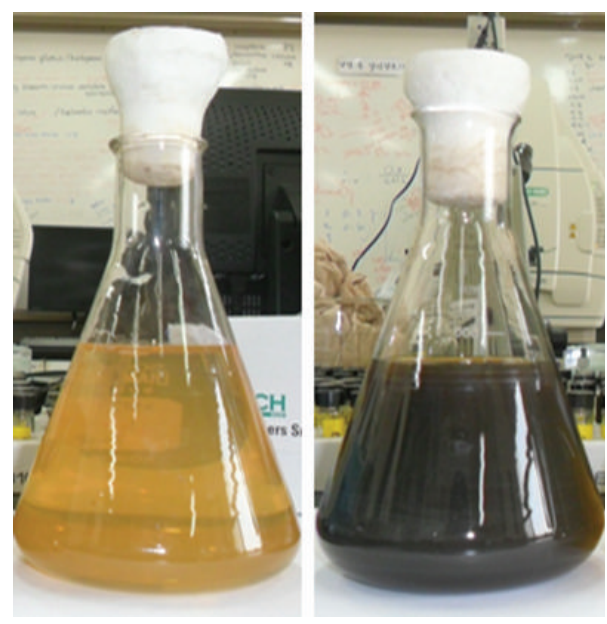

(a)

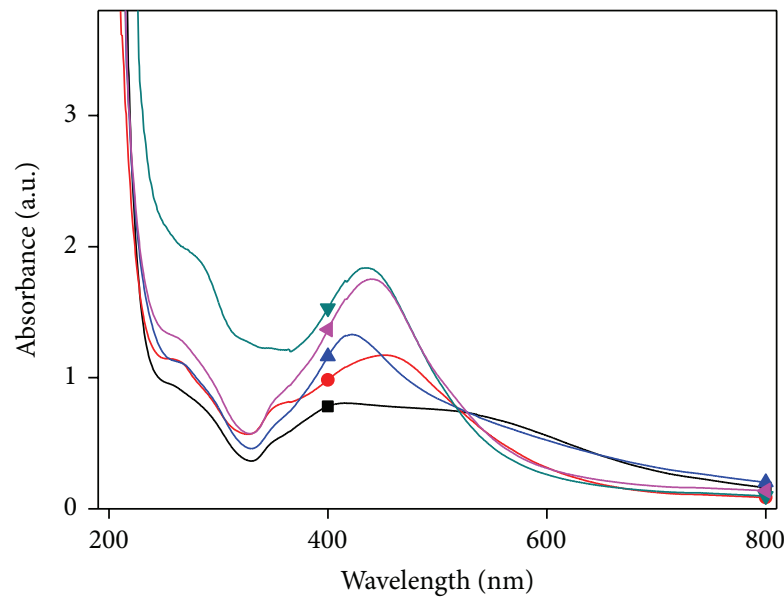

$\rightarrow$ - Buddleja officinalis/silver peak $434 \mathrm{~nm}$

$\rightarrow$ - Artemisia messer-schmidtiana/silver peak $421 \mathrm{~nm}$

—- Magnolia kobus/silver peak $416 \mathrm{~nm}$

$\rightarrow-$ Epimedium koreanum/silver peak $438 \mathrm{~nm}$

$\longleftarrow$ Mentha arvensis/silver peak $450 \mathrm{~nm}$

(b)

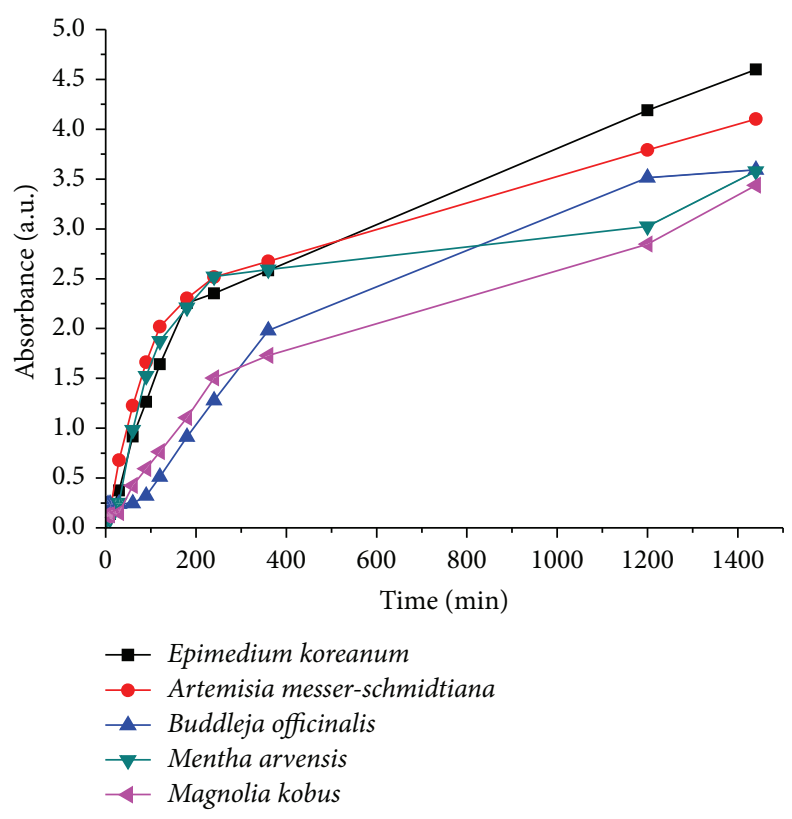

(c)

Figure 1: Synthesis of AgNPs by medicinal plant extracts by addition in a silver nitrate at $55^{\circ} \mathrm{C}$. (a) Flasks at left and right show reaction of 10 minutes and 24 hours after addition of plant extracts in $\mathrm{AgNO}_{3}$, (b) UV-visible spectra, and (c) time course of the synthesis of AgNPs by five plant extracts.

between 5 and $60 \mathrm{~nm}$ (Figure 4). The mean diameter of the synthesized AgNPs was found to be different for different plants (Figure 5). The average particle sizes for AgNPs synthesized by $M$. arvensis, B. officinalis, E. koreanum, A. messer-schmidtiana, and M. kobus were found to be 40.0 , $32.4,39.7,29.2$, and $24.7 \mathrm{~nm}$, respectively. The largest particle size was observed for $M$. arvensis and smallest for M. kobus (Figures 4 and 5). The resulting nanoparticle solutions were found to be stable for more than two months without agglomeration of particles.

A higher amount of glucose was produced in the presence of AgNPs in comparison with free cellulose (control). 
TABLE 1: Elemental composition in energy dispersive X-ray spectroscopy (EDS).

\begin{tabular}{|c|c|c|c|c|c|c|c|c|c|c|c|}
\hline \multirow{2}{*}{ Sample } & \multicolumn{11}{|c|}{ Element content (weight \%) } \\
\hline & $\mathrm{Al}$ & $\mathrm{Si}$ & $\mathrm{P}$ & S & $\mathrm{Cl}$ & $\mathrm{Ag}$ & $\mathrm{Pt}$ & $\mathrm{O}$ & $\mathrm{C}$ & $\mathrm{Zr}$ & Mo \\
\hline Mentha arvensis & 0.39 & 0.8 & 0.21 & 0.0 & 12.4 & 87.28 & - & - & - & - & - \\
\hline Buddleja officinalis & 0.61 & 0.11 & - & 0.08 & 4.09 & 94.15 & 0.97 & - & - & - & - \\
\hline Epimedium koreanum & 0.63 & 0.06 & - & - & 7.23 & 90.21 & 1.14 & 0.72 & - & - & - \\
\hline Artemisia messer-schmidtiana & 0.48 & - & - & - & 2.33 & 94.97 & 0.91 & - & 1.30 & - & - \\
\hline \multirow[t]{2}{*}{ Magnolia kobus } & 0.45 & - & - & - & 0.50 & 97.87 & - & - & - & 088 & 0.30 \\
\hline & \multicolumn{11}{|c|}{ Element content (atom \%) } \\
\hline Mentha arvensis & 1.24 & 0.23 & 0.58 & 0.01 & 28.95 & 68.98 & - & - & - & - & - \\
\hline Buddleja officinalis & 2.20 & 0.38 & - & 0.23 & 11.30 & 85.41 & 0.49 & - & - & - & - \\
\hline Epimedium koreanum & 2.08 & 0.20 & - & - & 18.27 & 74.88 & 0.52 & 4.05 & - & - & - \\
\hline Artemisia messer-schmidtiana & 1.67 & - & - & - & 6.10 & 81.73 & 0.43 & - & 10.07 & - & - \\
\hline Magnolia kobus & 1.77 & - & - & - & 1.47 & 95.42 & - & - & - & 1.01 & 0.33 \\
\hline
\end{tabular}

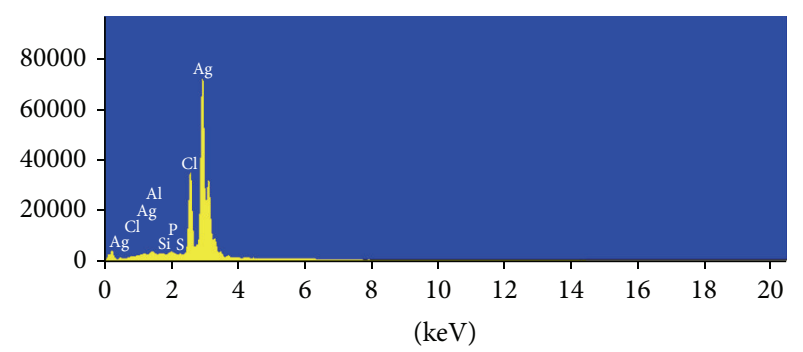

(a)

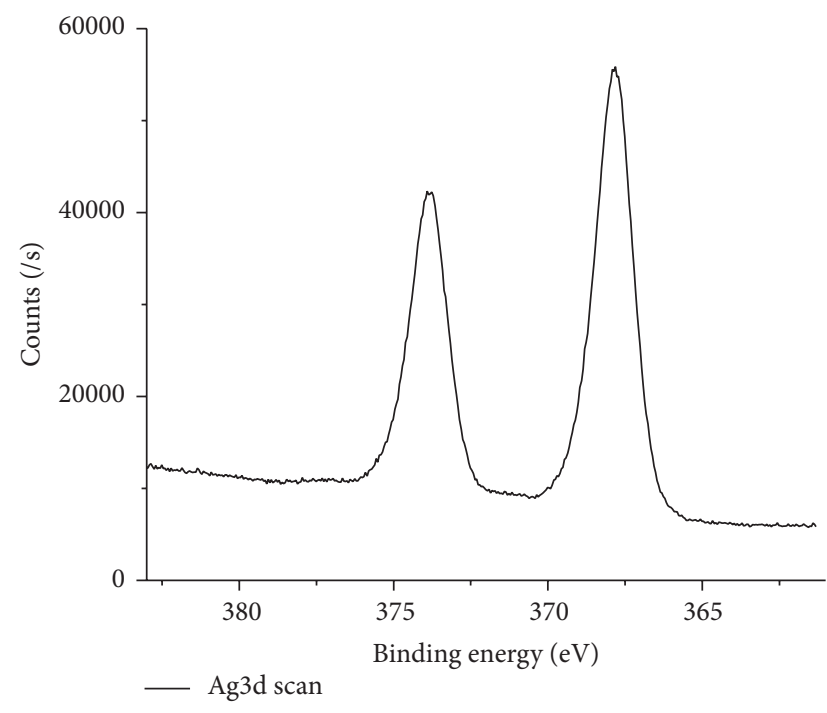

(b)

FIGURE 2: A representative (a) energy dispersive X-ray spectroscopy (EDS) and (b) X-ray photoelectron spectroscopy (XPS) image of the AgNPs synthesized by medicinal plant extracts of Mentha arvensis var. piperascens.

The hydrolysis efficiency of AgNPs showed the following order: M. kobus > A. messer-schmidtiana > B. officinalis > E. koreanum $>M$. arvensis (Figure 6). The reducing sugar formation increased by about twofold for $M$. kobus over control, suggesting a significant role of nanoparticles in acting as nanocatalyst for enhanced degradation of cellulose to reducing sugars. The rate of reaction was found to be increased in the presence of AgNPs and is depicted in Figure 6. The results of reducing sugar assay suggested an increased enzyme activity in cellulose hydrolysis.

The exact mechanism behind the enhancement of cellulose hydrolysis by AgNPs is not clear at this juncture but AgNPs may be acting as catalyst by some unknown mechanism, which needs further detailed investigation. Interestingly, plant synthesized AgNPs of smaller size displayed better catalytic activity than larger sized AgNPs. AgNPs with smaller size have been known to display better bioactivities than larger particles [2]. There may be different possibilities for the faster reaction rate of cellulose hydrolysis in the presence of AgNPs. One of the possible scenarios can be explained as follows. Plant metabolites reduce silver nitrate to AgNPs. The interaction of cellulase with cellulose and plant synthesized AgNPs leads to the immobilization of cellulase on the surface of the AgNPs and degradation of cellulose by the enzyme into reducing sugars. AgNPs generally have the tendency to agglomerate faster in any biological medium and sediment at the bottom. However, such agglomeration was not observed during the reaction, indicating that the AgNPs have the chances of being stabilized by the protein molecule through the thiol linkages, and thus the enzyme 


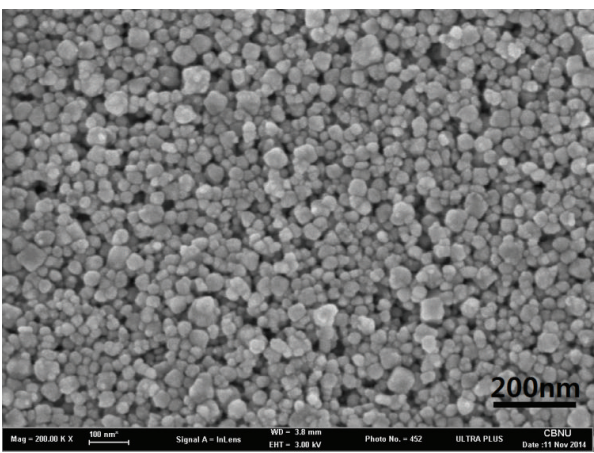

(a)

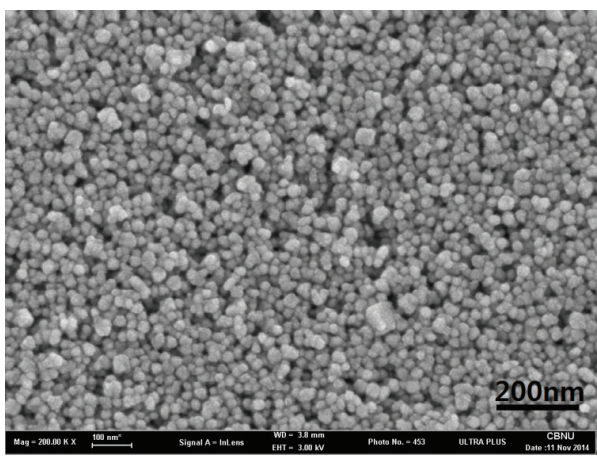

(c)

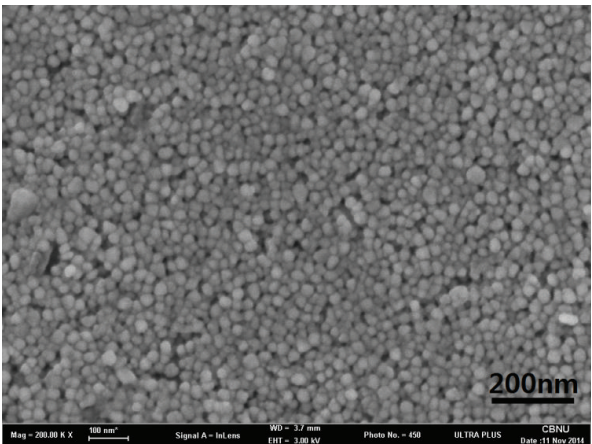

(b)

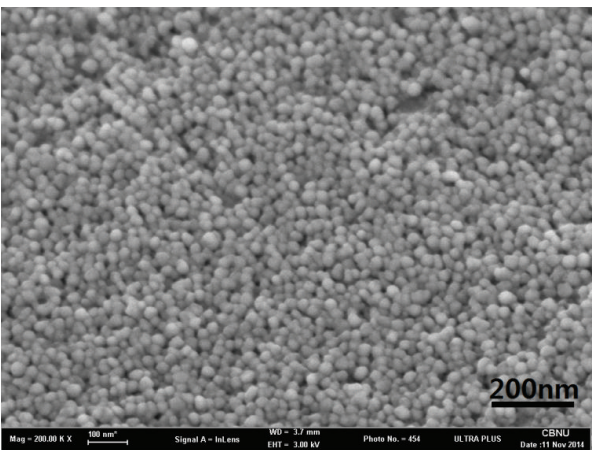

(d)

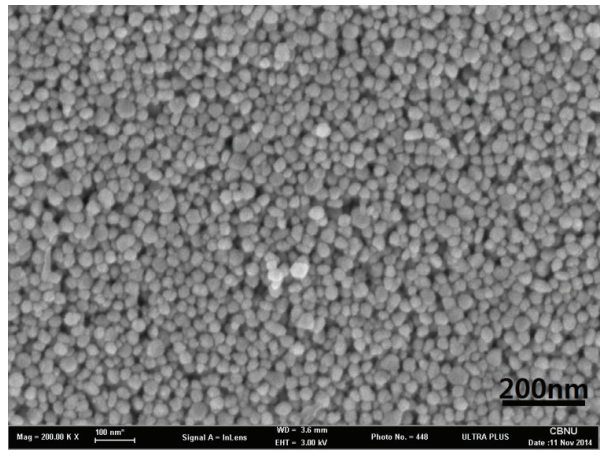

(e)

FIGURE 3: Scanning electron microscopy (SEM) of the AgNPs synthesized by five medicinal plant extracts: (a) Mentha arvensis var. piperascens, (b) Buddleja officinalis Maximowicz, (c) Epimedium koreanum Nakai, (d) Artemisia messer-schmidtiana Besser, and (e) Magnolia kobus.

molecule might be immobilized as suggested by Deka et al. [16]. The efficiency of the enzyme increases on solid support as compared to its free form [17]. The degradation in this situation occurs at a higher rate because the collision produced by the system would be less than when compared to free cellulose. AgNPs could possibly be acting as nanocatalyst in the hydrolysis of cellulose catalyzed by cellulase and increase the reaction rate although the exact binding mechanism remains to be explored.

In the present study, cellulose and plant synthesized AgNPs after interaction with cellulase were capable of breaking down the cellulose complex with the attachment of the enzyme over its surface thereby being immobilized and degrading cellulose much faster than when compared to free cellulose. As the collision frequency between the free enzyme and the substrate molecule and their steric orientations forms the basis of the enzyme activity, the constraint was overcome by the immobilized enzyme with the support of a solid nanoparticle whereas it did not occur in the case of free cellulose similar to reported by Rangnekar et al. [18]. Therefore, the reaction rate was probably high, and the breakdown of cellulose to smaller molecules like monosaccharides and disaccharides was faster. The AgNPs were found to be better nanocatalysts compared 


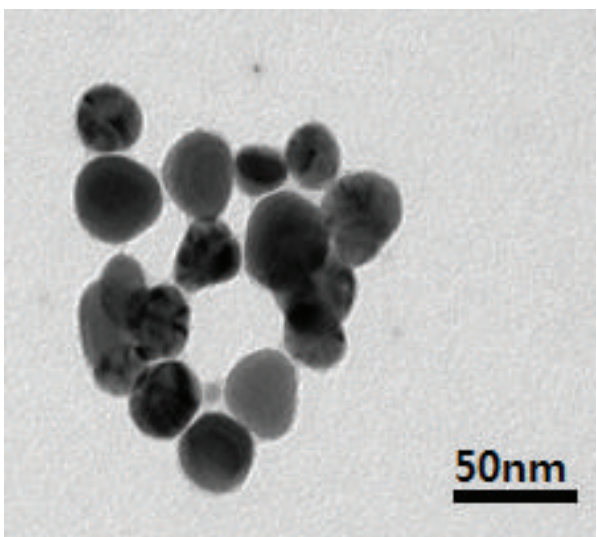

(a)

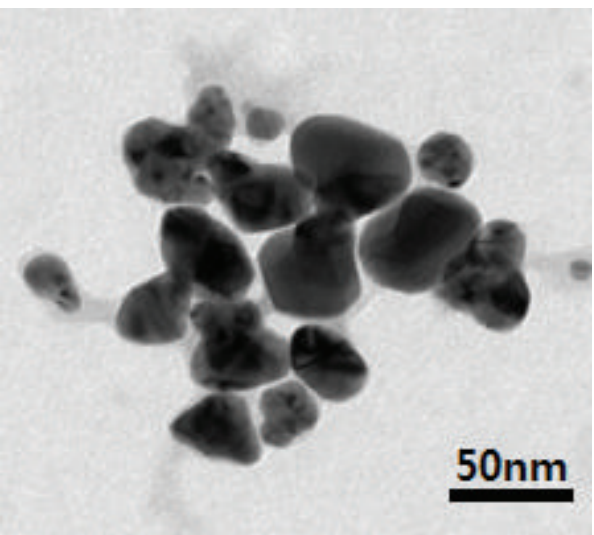

(c)

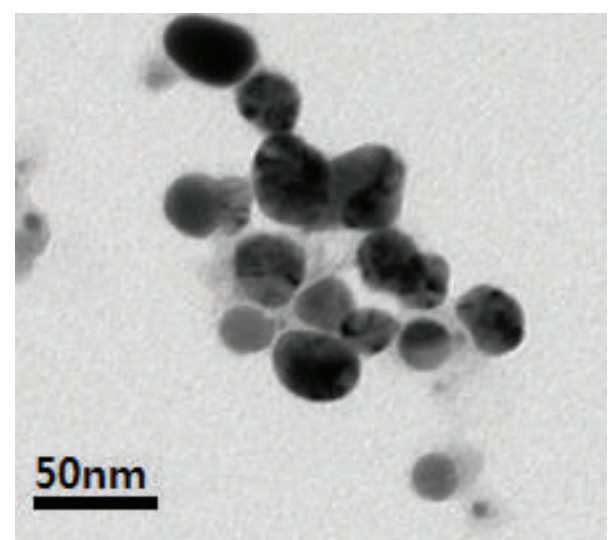

(b)

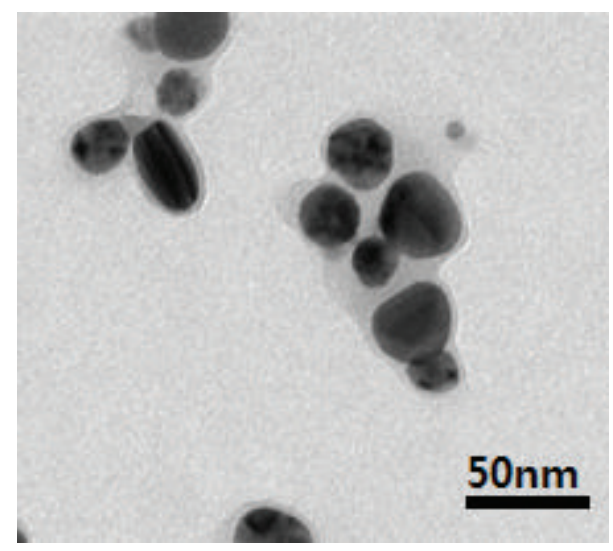

(d)

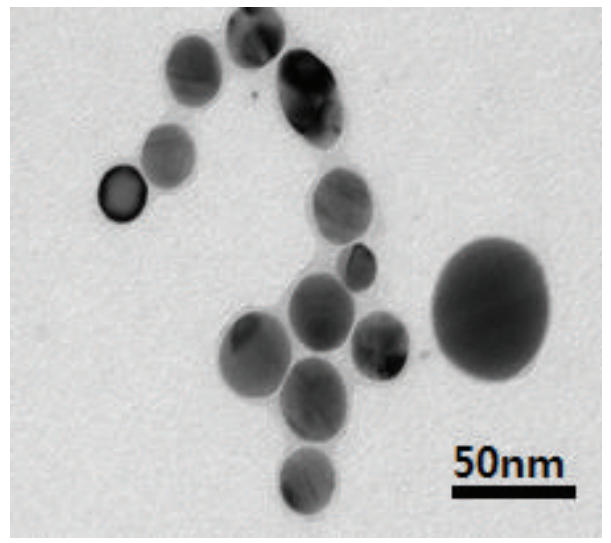

(e)

FIGURE 4: Transmission electron microscopy (TEM) images of the AgNPs synthesized by five medicinal plant extracts (a) Mentha arvensis var. piperascens, (b) Buddleja officinalis Maximowicz, (c) Epimedium koreanum Nakai, (d) Artemisia messer-schmidtiana Besser, and (e) Magnolia kobus.

to gold nanoparticles in degrading starch at a faster rate $[16,18]$.

\section{Conclusion}

The plant synthesized AgNPs showed an increased rate of reaction with cellulase compared with free cellulose. The degradation of cellulose in the presence of AgNPs rapidly produced larger amounts of reducing sugars. AgNPs obtained with M. kobus showed highest reducing sugar formation. This study demonstrates that the nanoparticles may be useful in the field of nanocatalysis such as rapid degradation of the complex molecules to simpler ones by immobilizing the enzymes onto the surface of nanoparticles and different industrial applications. They can also be useful in developing assay kits for sensing and biomedical applications. 


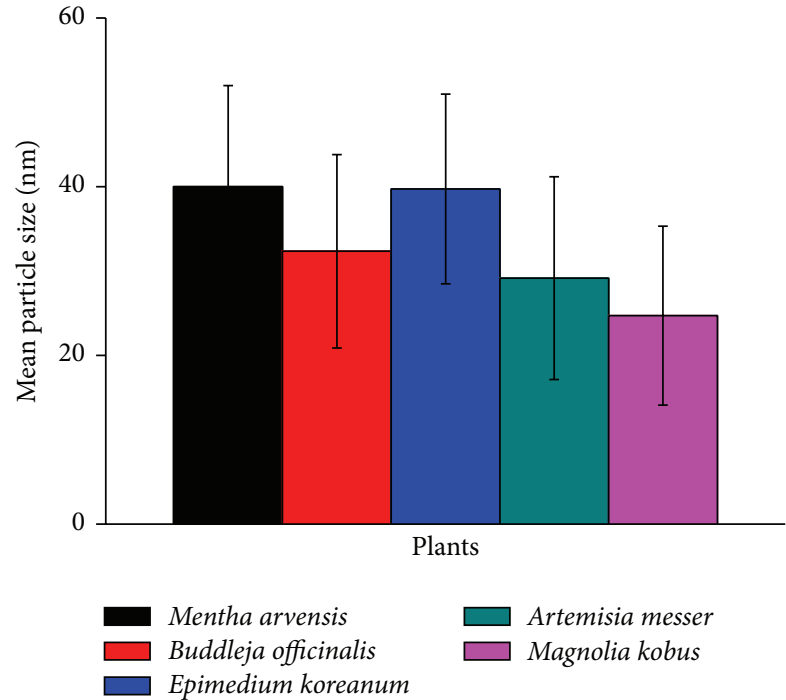

FIGURE 5: Mean particle size histogram of the AgNPs synthesized by five medicinal plant extracts.

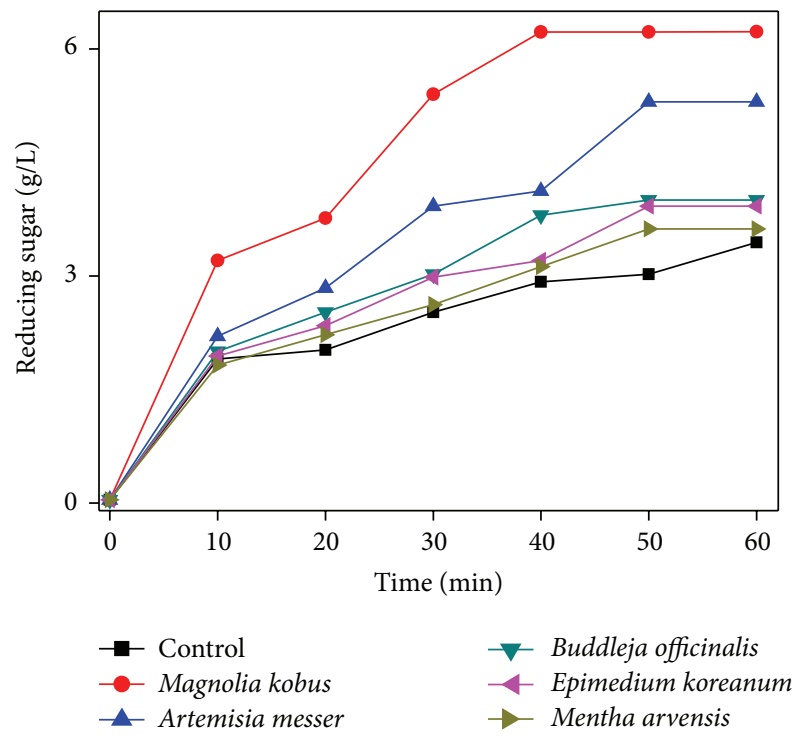

Figure 6: Comparative study of cellulose degradation at $25^{\circ} \mathrm{C}$ in the presence of AgNPs and free cellulose (control).

\section{Conflict of Interests}

The authors declare that there is no conflict of interests regarding the publication of this paper.

\section{Acknowledgment}

This research was supported by the Basic Science Research Program of the National Research Foundation of Korea (NRF) funded by the Ministry of Education, Science and Technology (NRF-2012R1A1A2006375 and NRF2013R1A2A2A01067117).

\section{References}

[1] Q. H. Tran, V. Q. Nguyen, and A.-T. Le, "Silver nanoparticles: synthesis, properties, toxicology, applications and perspectives," Advances in Natural Sciences: Nanoscience and Nanotechnology, vol. 4, no. 3, Article ID 033001, 2013.

[2] H. P. Borase, B. K. Salunke, R. B. Salunkhe et al., "Plant extract: a promising biomatrix for ecofriendly, controlled synthesis of silver nanoparticles," Applied Biochemistry and Biotechnology, vol. 173, no. 1, pp. 1-29, 2014.

[3] P. P. Gan and S. F. Y. Li, "Potential of plant as a biological factory to synthesize gold and silver nanoparticles and their applications," Reviews in Environmental Science and Biotechnology, vol. 11, no. 2, pp. 169-206, 2012.

[4] H. Bar, D. K. Bhui, G. P. Sahoo, P. Sarkar, S. P. De, and A. Misra, "Green synthesis of silver nanoparticles using latex of Jatropha curcas," Colloids and Surfaces A: Physicochemical and Engineering Aspects, vol. 339, no. 1-3, pp. 134-139, 2009.

[5] S. A. Babu and H. G. Prabu, "Synthesis of AgNPs using the extract of Calotropis procera flower at room temperature," Materials Letters, vol. 65, no. 11, pp. 1675-1677, 2011.

[6] J. Y. Song and B. S. Kim, "Rapid biological synthesis of silver nanoparticles using plant leaf extracts," Bioprocess and Biosystems Engineering, vol. 32, no. 1, pp. 79-84, 2009.

[7] N. Basavegowda, A. Idhayadhulla, and Y. R. Lee, “Tyrosinase inhibitory activity of silver nanoparticles treated with Hovenia dulcis fruit extract: an in vitro study," Materials Letters, vol. 129, pp. 28-30, 2014.

[8] D. Raju, R. K. Vishwakarma, B. M. Khan, U. J. Mehta, and A. Ahmad, "Biological synthesis of cationic gold nanoparticles and binding of plasmid DNA," Materials Letters, vol. 129, pp. 159-161, 2014.

[9] X. Ren, X. Meng, D. Chen, F. Tang, and J. Jiao, "Using silver nanoparticle to enhance current response of biosensor," Biosensors and Bioelectronics, vol. 21, no. 3, pp. 433-437, 2005.

[10] H. Cong and J. A. Porco, "Chemical synthesis of complex molecules using nanoparticle catalysis," ACS Catalysis, vol. 2, no. 1, pp. 65-70, 2012.

[11] D. Klemm, B. Heublein, H.-P. Fink, and A. Bohn, "Cellulose: fascinating biopolymer and sustainable raw material," Angewandte Chemie International Edition, vol. 44, no. 22, pp. 33583393, 2005.

[12] A. Saxena, A. Kumar, and S. Mozumdar, "Ni-nanoparticles: an efficient green catalyst for chemo-selective oxidative coupling of thiols," Journal of Molecular Catalysis A: Chemical, vol. 269, no. 1-2, pp. 35-40, 2007.

[13] J. Yan, H. Tao, M. Zeng et al., "PVP-capped silver nanoparticles as catalyst for oxidative coupling of thiols to disulfides," Chinese Journal of Catalysis, vol. 30, no. 9, pp. 856-858, 2009.

[14] H. Cong, C. F. Becker, S. J. Elliott, M. W. Grinstaff, and J. A. Porco Jr., "Silver nanoparticle-catalyzed Diels-Alder cycloadditions of $2^{\prime}$-hydroxychalcones," Journal of the American Chemical Society, vol. 132, no. 21, pp. 7514-7518, 2010.

[15] V. Ernest, P. J. Shiny, A. Mukherjee, and N. Chandrasekaran, "Silver nanoparticles: a potential nanocatalyst for the rapid degradation of starch hydrolysis by $\alpha$-amylase," Carbohydrate Research, vol. 352, pp. 60-64, 2012.

[16] J. Deka, A. Paul, A. Ramesh, and A. Chattopadhyay, "Probing $\mathrm{Au}$ nanoparticle uptake by enzyme following the digestion of a starch-Au-nanoparticle composite," Langmuir, vol. 24, no. 18, pp. 9945-9951, 2008. 
[17] Z.-J. Jiang, C.-Y. Liu, and L.-W. Sun, "Catalytic properties of silver nanoparticles supported on silica spheres," The Journal of Physical Chemistry B, vol. 109, no. 5, pp. 1730-1735, 2005.

[18] A. Rangnekar, T. K. Sarma, A. K. Singh, J. Deka, A. Ramesh, and A. Chattopadhyay, "Retention of enzymatic activity of $\alpha$-amylase in the reductive synthesis of gold nanoparticles," Langmuir, vol. 23, no. 10, pp. 5700-5706, 2007. 

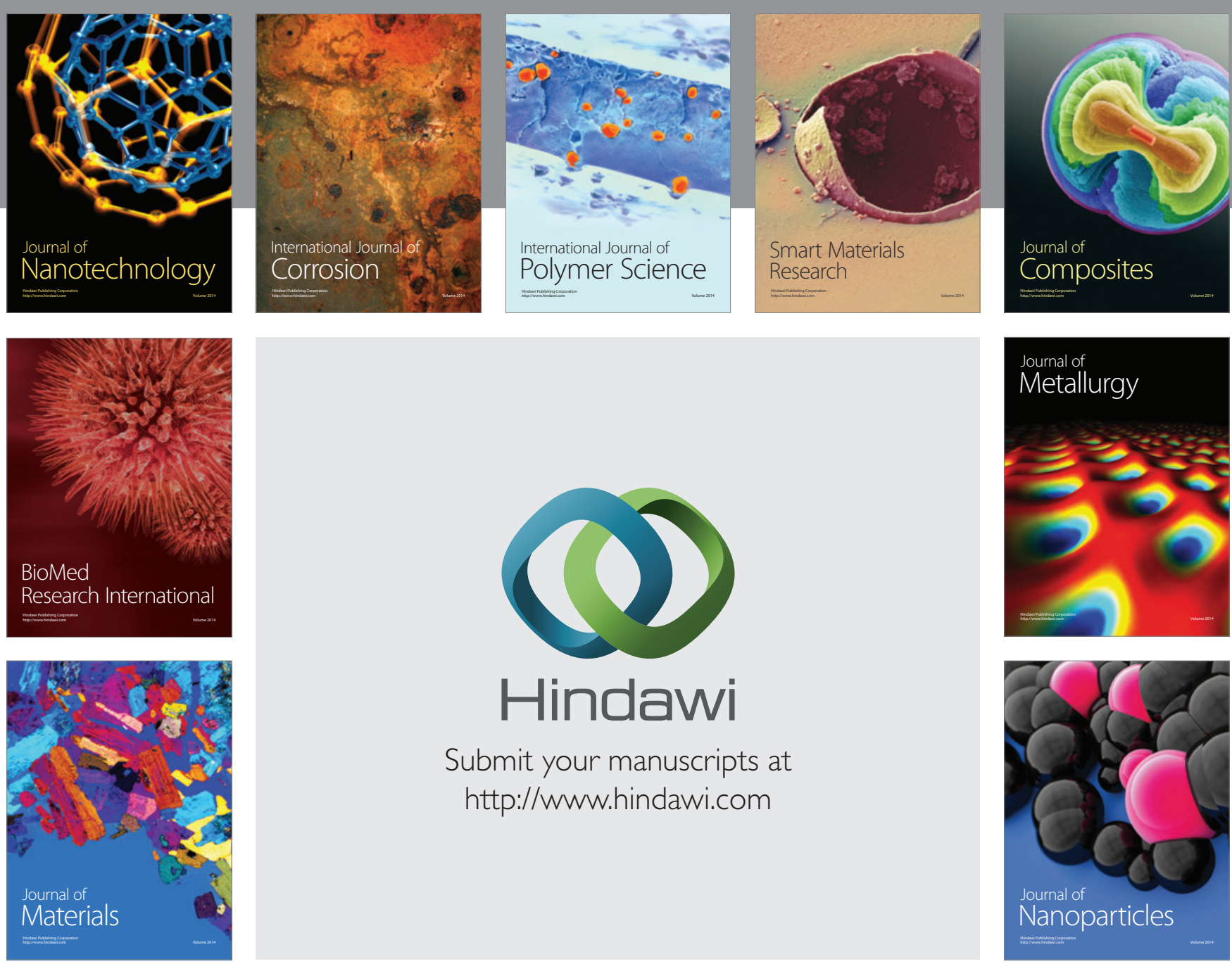

Submit your manuscripts at http://www.hindawi.com
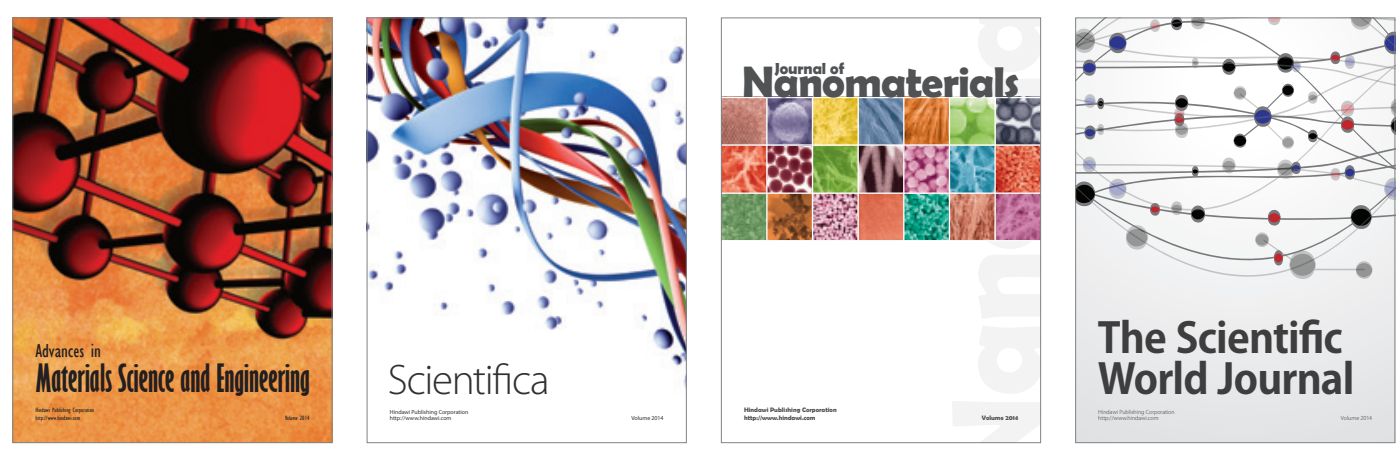

\section{The Scientific World Journal}
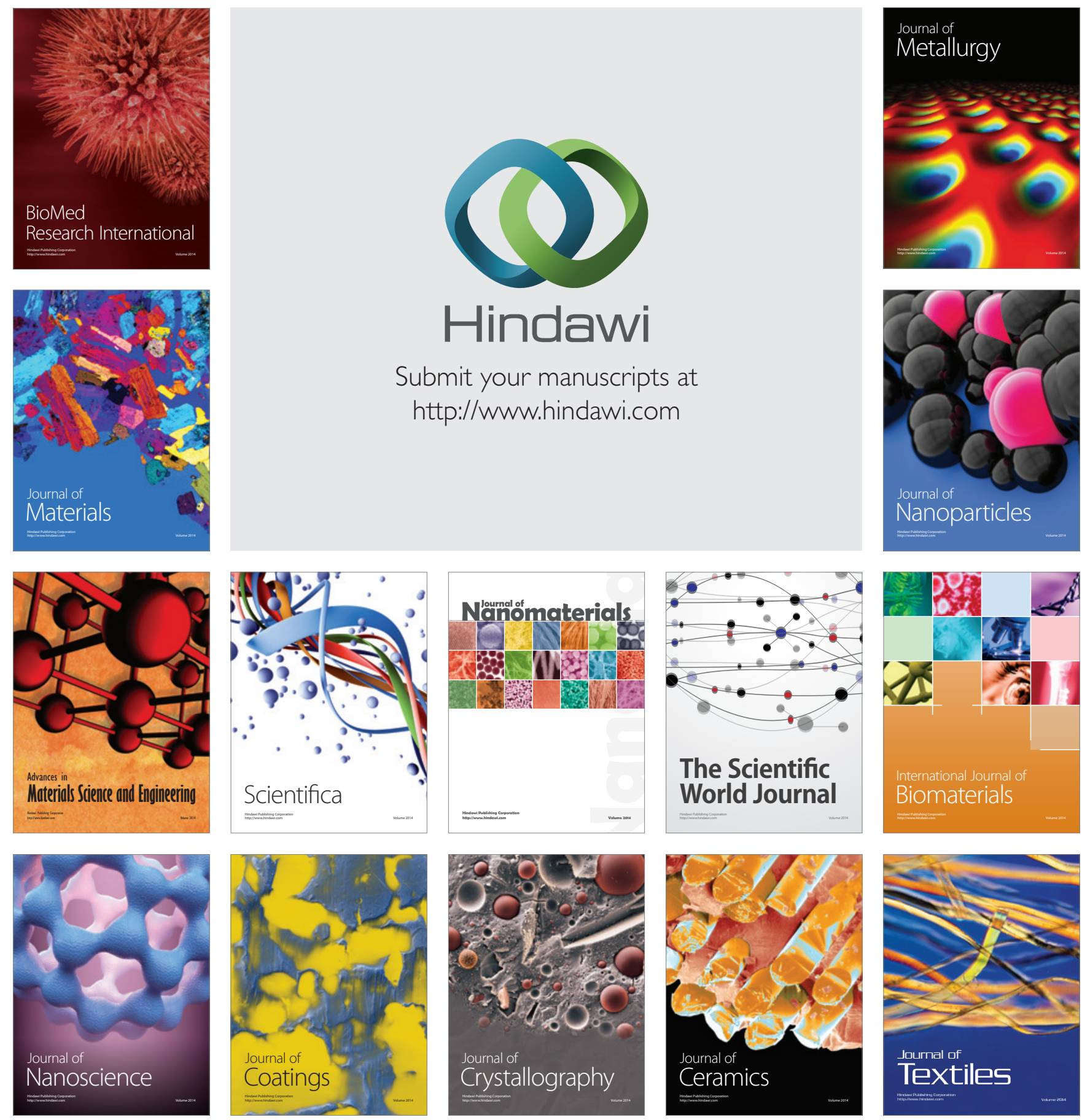